\title{
Assessment of normal tricuspid valve anatomy in adults by real-time three-dimensional echocardiography
}

\author{
Ashraf M. Anwar - Marcel L. Geleijnse - Osama I. I. Soliman • \\ Jackie S. McGhie · René Frowijn - Attila Nemes • \\ Annemien E. van den Bosch · Tjebbe W. Galema • \\ Folkert J. ten Cate
}

Received: 26 December 2006/ Accepted: 29 January 2007 / Published online: 23 February 2007

(C) Springer Science+Business Media B.V. 2007

\begin{abstract}
Background The tricuspid valve (TV) is a complex structure. Unlike the aortic and mitral valve it is not possible to visualize all TV leaflets simultaneously in one cross-sectional view by standard two-dimensional echocardiography (2DE) either transthoracic or transesophageal due to the position of TV in the far field.

Aim Quantitative and qualitative assessment of the normal TV using real-time 3-dimensional echocardiography (RT3DE).

Methods RT3DE was performed for 100 normal adults (mean age $30 \pm 9$ years, 65\% males). RT3DE visualization was evaluated by 4-point score (1: not visualized, 2: inadequate, 3: sufficient, and 4: excellent). Measurements included TV annulus diameters (TAD), TV area (TVA), and commissural width.

Results In $90 \%$ of patients with good $2 \mathrm{DE}$ image quality, it was possible to analyse TV
\end{abstract}

A. M. Anwar · M. L. Geleijnse - O. I. I. Soliman ·

J. S. McGhie - R. Frowijn · A. Nemes ·

A. E. van den Bosch · T. W. Galema .

F. J. ten Cate $(\square)$

The Thoraxcenter, Erasmus MC, Dr. Molewaterplein 40, Rotterdam 3015 GD, The Netherlands

e-mail: f.j.tencate@erasmusmc.nl

A. M. Anwar · O. I. I. Soliman

Department of Cardiology, Al-Husein University

Hospital, Al-Azhar University, Cairo, Egypt anatomy by RT3DE. A detailed anatomical structure including unique description and measurement of tricuspid annulus shape and size, TV leaflets shape, and mobility, and TV commissural width were obtained in majority of patients. Identification of each TV leaflet as seen in the routine $2 \mathrm{DE}$ views was obtained.

Conclusion RT3DE of the TV is feasible in a large number of patients. RT3DE may add to functional $2 \mathrm{DE}$ data in description of TV anatomy and providing highly reproducible and actual reality (anatomical and functional) measurements.

Keywords Normal tricuspid valve - Tricuspid valve anatomical structure $\cdot$ Real-Time threedimensional echocardiography

\section{Introduction}

The tricuspid valve (TV) is a multi-component complex structure [1]. In classic anatomic studies the anterior, septal and posterior TV cusps were described $[2,3]$. Unlike the aortic and mitral valve it is not possible to visualize all TV cusps simultaneously in one cross-sectional view by standard transthoracic two-dimensional echocardiography (2DE) [4]. During transesophageal 2DE small changes in transducer angle, probe position and rotation may bring to light some 
additional TV details [5, 6]. However, because of the position of the TV in the far field in relation to probe, transesophageal 2DE can still only provide limited information and can also not visualize all TV cusps simultaneously. In three-dimensional (3D) transesophageal image reconstruction and intracardiac echocardiography studies this goal could be achieved but at the cost of some procedural risks and an increase in procedural duration [7, 8]. Real-time three-dimensional echocardiography (RT3DE) can visualize the atrio-ventricular valves from both the ventricular and atrial side in detail without these limitations [9]. This study aimed to apply RT3DE for quantitative and qualitative assessment of normal TV anatomy.

\section{Subjects and methods}

In one hundred patients (mean age $30 \pm 9$ years, $65 \%$ males) the TV was examined by transthoracic RT3DE after an informed consent. All patients had sinus rhythm and normal right-sided heart (normal right ventricular dimensions and function, normal right atrial dimension, trivial or absent tricuspid regurgitation and normal tricuspid valve function). Patients with good
2DE image quality only were included. RT3DE was done with a commercially available ultrasound system

(Philips Sonos 7500, Best, The Netherlands) attached to a X4 matrix array transducer capable of providing real-time B-mode images. The 3D data set was collected within approximately 5-10 s of breath holding in full volume mode from an apical window and transferred for off-line analysis with TomTec software (Unterschleissheim, Munich, Germany). Data analysis of 3D images was based on a two-dimensional approach relying on images obtained initially from the apical 4-chamber view. The images were adjusted to put the TV in the center of interest. To exclude non-relevant tissue, the TV was sliced between the two narrowest lines by which all parts of the TV leaflets were still in between. The TomTec software allows in this way visualization of the short-axis TV view in a 3D display (see Fig. 1). RT3DE gain and brightness were adjusted to improve delineation of anatomic structures. The following points were checked for visualization: 1) tricuspid annulus diameter and area, 2) TV leaflets (number, mobility, thickness and relation to each other), 3) TV area, and 4) TV commissures (antero-septal, antero-posterior, and postero-septal) including the position of their closure
Fig. 1 TomTec quadri screen display of the tricuspid valve. The upper two images represent twodimensional views created from the $3 \mathrm{D}$ data set (4-chamber, left and orthogonal view, right). The lower left image represents a twodimensional short axis view and the lower right image represents the $3 \mathrm{D}$ image

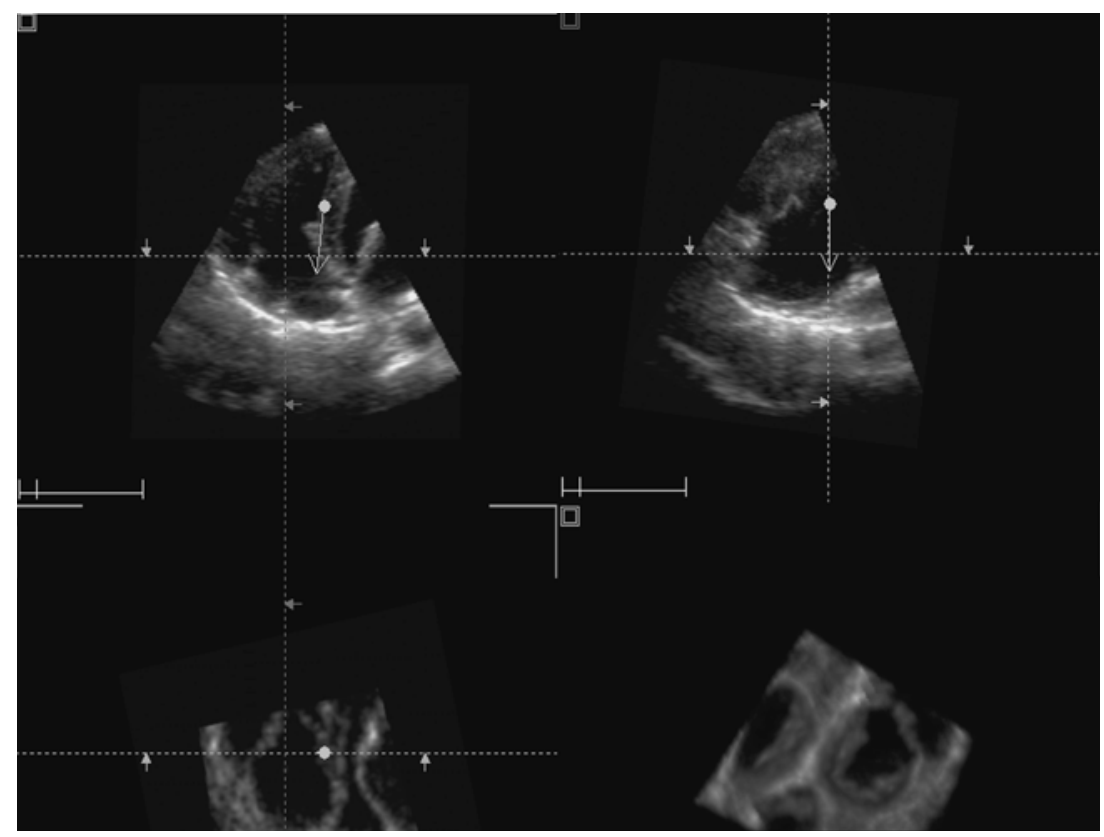


lines. All these structures were classified according to a subjective 4-point scale for image quality $(1=$ not visualized, $2=$ inadequate, $3=$ sufficient and 4 = good).

For quantitative assessment of TV the following RT3DE data were obtained: 1) TV annulus diameter defined as the widest diameter that could be measured from an end-diastolic still frame, 2) maximal TV annulus area obtained from an end-diastolic still frame and measured by manual planimetry, 3) TV area defined as the narrowest part of the TV at the time of maximal opening and measured by manual planimetry, and 4) TV commissural width obtained from a latediastolic still frame using zoom function to avoid underestimation. The images were optimized for each commissure along its plane to measure the maximal width of the angle formed by the two adjacent TV leaflets.

To identify the TV leaflets visualized by the standard 2DE images the TomTec quad screen display was used. As seen in Fig. 1, this screen contains four images; the upper two images are 2DE images perpendicular to each other, the lower two images are a short-axis 2DE image and a RT3DE image. From the properly chosen twodimensional image a mid-diastolic frame was selected to visualize the TV leaflets just separated from each other. Each leaflet was defined by a marker, after which this marker position was compared with the RT3DE image to detect which leaflet was shown in the 2DE images. Analysis of images was done by two experienced echocardiographers (AMA, JSM) independently. Each one dealt with the full volume image as acquired from echo machine and the selection of cut plane, angulation and gain setting adjustment were dependable on his experience.

\section{Statistical analysis}

All data obtained by RT3DE were presented as mean \pm SD. Interobserver and intraobserver agreements for the visualization score were estimated using kappa values for each morphologic feature and classified as poor (kappa <0.4), moderate (kappa 0.4 to 0.7 ), or good (kappa $>0.7)$. Interobserver and intraobserver variabilities for RT3DE measurements was assessed according to the Bland and Altman method in a randomly selected group of 50 patients [10] Table 1.

\section{Results}

Acquisition and analysis of the RT3DE data was performed in approximately $10 \mathrm{~min}$ per patient. The TV could be visualized in $90 \%$ of patients enface from both ventricular and atrial aspects in relation to adjacent cardiac structures. In these 90 patients detailed analysis of the TV was performed including tricuspid annulus shape and size, TV leaflets shape, size, and mobility, and commissural width.

Tricuspid annulus

Tricuspid annulus visualization was good in 54 patients $(60 \%)$, sufficient in 27 patients $(30 \%)$, and inadequate in 9 patients $(10 \%)$. As seen in Fig. 2, tricuspid annulus shape appeared as oval rather than circular. Tricuspid annulus diameter and area could be measured in 63 patients $(70 \%)$, normal values were $4.0 \pm 0.7 \mathrm{~cm}$ and $10.0 \pm$ $2.9 \mathrm{~cm}^{2}$, respectively.

Tricuspid valve leaflets

Visualization of the three TV leaflets (in motion) was good in 72 patients $(80 \%)$, sufficient in 9 patients $(10 \%)$, and inadequate in another 9 patients $(10 \%)$. The anterior leaflet was the

Table 1 Scores for real-time three-dimensional echocardiography visualization of TV structures

\begin{tabular}{lllll}
\hline Score & $\begin{array}{l}\text { TV } \\
\text { annulus }\end{array}$ & $\begin{array}{l}\text { TV } \\
\text { leaflets }\end{array}$ & $\begin{array}{l}\text { TV } \\
\text { area }\end{array}$ & $\begin{array}{l}\text { TV } \\
\text { commissures }\end{array}$ \\
\hline $\begin{array}{l}\text { Good (4) } \\
\text { Sufficient (3) }\end{array}$ & $\begin{array}{l}60 \% \\
30 \%\end{array}$ & $\begin{array}{l}80 \% \\
10 \%\end{array}$ & $\begin{array}{l}55 \% \\
30 \%\end{array}$ & $\begin{array}{l}50 \% \\
20 \%\end{array}$ \\
$\begin{array}{l}\text { Inadequate } \\
\quad(2)\end{array}$ & $10 \%$ & $10 \%$ & $15 \%$ & $20 \%$ \\
$\begin{array}{l}\text { Not } \\
\quad \text { visualized }\end{array}$ & $0 \%$ & $0 \%$ & $0 \%$ & $10 \%$ \\
$\quad(1)$ & & & & \\
$\begin{array}{l}\text { Mean score } \\
\text { Median score }\end{array}$ & $3.5 \pm 0.7$ & $3.7 \pm 0.6$ & $3.4 \pm 0.7$ & $3.1 \pm 1.0$ \\
\hline
\end{tabular}

Abbreviations: $\mathrm{TV}=$ tricuspid valve 
Fig. 2 Oval-shaped Tricuspid annulus (the line represent the tricuspid annulus diameter, the dots demark the area)

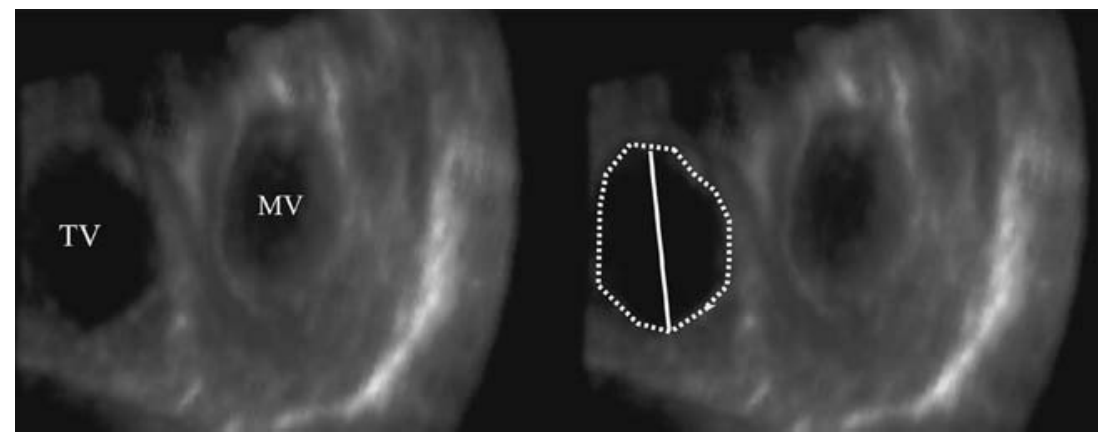

largest and most mobile of the three leaflets and had a nearly semicircular shape. The septal leaflet was the least mobile and had a semi-oval shape. Its position was parallel to the interventricular septum. The posterior leaflet was the smallest one with variable shape. It was clearly separated from the septal leaflet in all patients but in $10 \%$ of patients it was hard to discriminate the posterior leaflet from the anterior leaflet even during maximal TV opening.

From the RT3DE data set all standard twodimensional TV cross-sections (apical 4-chamber, parasternal short-axis and parasternal long-axis right ventricular inflow) were simulated. As seen in Fig. 3, in the apical 4-chamber view in all patients the septal leaflet was seen adjacent to the septum and the anterior leaflet was seen adjacent to the right ventricular free wall. In the parasternal short-axis view, the posterior leaflet was seen adjacent to the right ventricular free wall in $92 \%$ of patients and in the remaining $8 \%$ no leaflet could be obtained although modification of the cut plane downward could identify this leaflet. In this view the leaflet adjacent to the aorta was the anterior in $52 \%$ and the septal leaflet in $48 \%$. In the parasternal right ventricular inflow view the leaflets seen were identical to the apical 4-chamber view with in all patients the septal leaflet seen adjacent to the septum and the anterior leaflet seen adjacent to the right ventricular free wall.

\section{Tricuspid valve area}

Visualization of the triangular shaped TV area was good in 50 patients (55\%), sufficient in 27 patients $(30 \%)$, and inadequate in 13 patients $(15 \%)$. As seen in Fig. 4, the anterior and septal leaflets formed the TV area's angle and the small posterior leaflet formed its base. TV area
Fig. 3 Identification of the tricuspid valve leaflets seen on two-dimensional imaging. Below the 2D images, percentage of leaflet identification in each standard view depending the RT3DE images

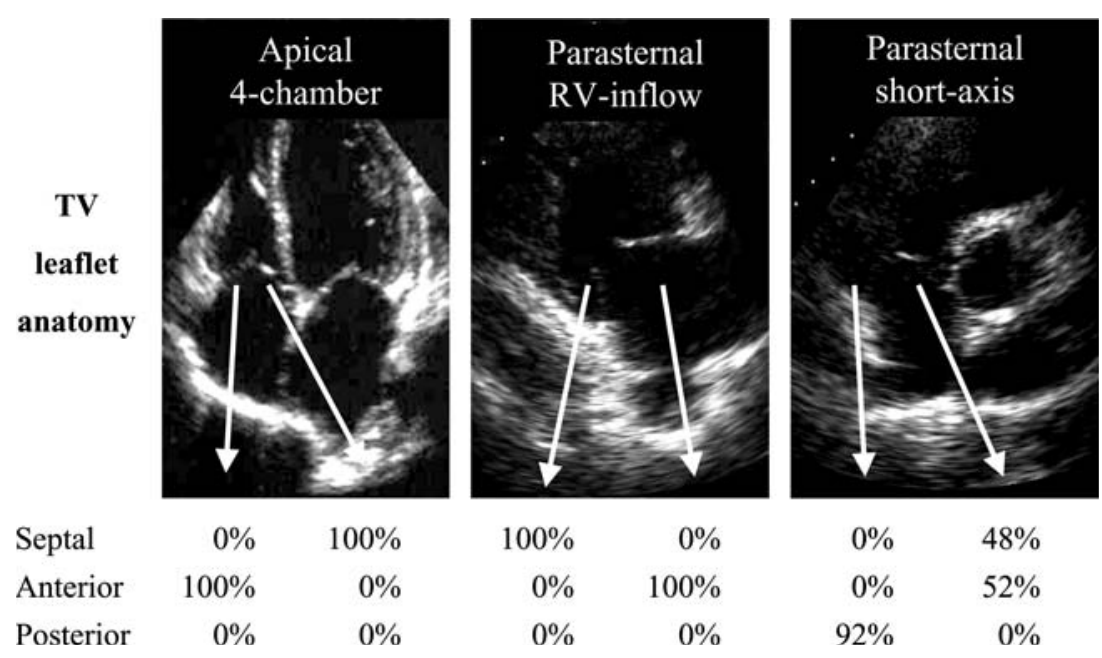


Fig. 4 Triangular shape TV area and commissural views

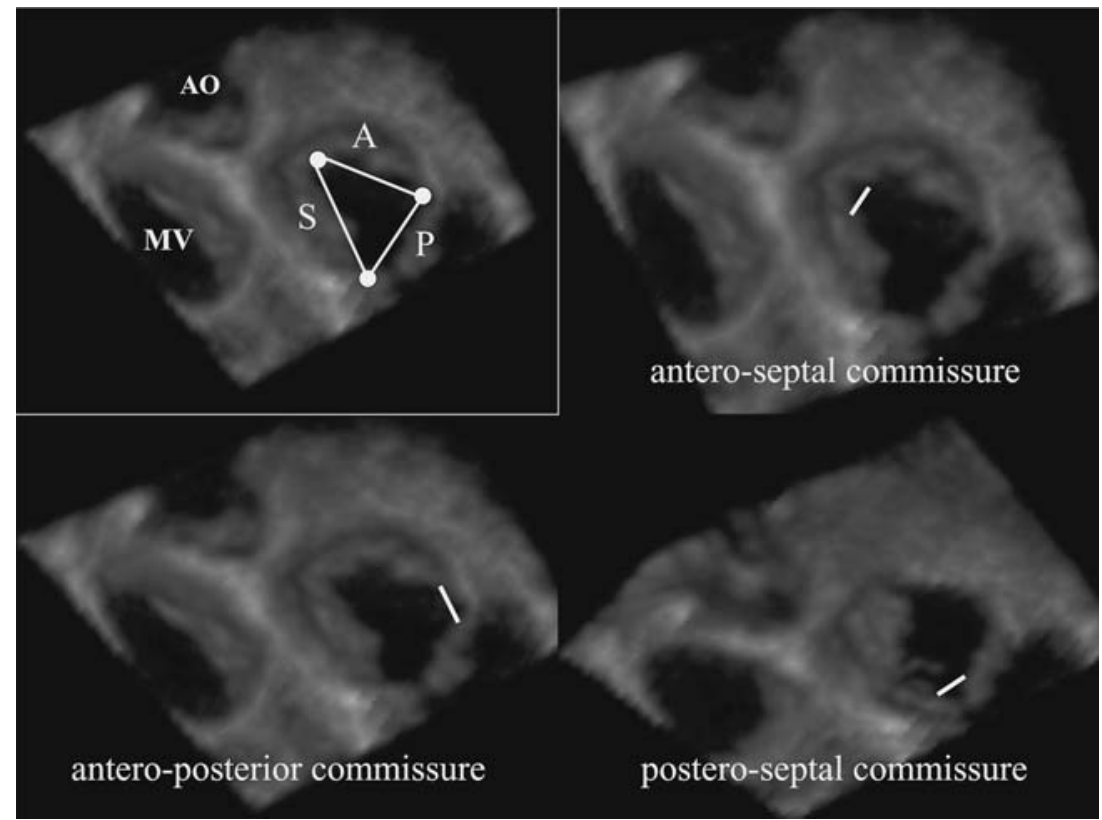

could be measured in 77 patients $(86 \%)$ and mean TV area in these patients was $4.8 \pm 1.6 \mathrm{~cm}^{2}$.

Tricuspid valve commissures

As seen in Fig. 4, the three TV leaflets were separated from each other by three commissures. The commissures and the direction of closure lines were good visualized in 45 patients $(50 \%)$, sufficient in 18 patients $(20 \%)$, inadequate in 18 patients $(20 \%)$, and not visualized in 9 patients $(10 \%)$. TV commissural width could be obtained in 63 patients $(70 \%)$, mean commissural width in these patients was $5.4 \pm 1.5 \mathrm{~mm}$ for the anteroseptal commissure, $5.2 \pm 1.5 \mathrm{~mm}$ for the posteroseptal commissure, and $5.1 \pm 1.1 \mathrm{~mm}$ for the antero-posterior commissure, respectively. Visualization and measurement of commissures was relatively easy for the antero-septal commissure and most difficult for the antero-posterior commissure. All measurements are listed in Table 2 as absolute value and indexed to body surface area.

Interobserver variability

The visualization score between two observers was good for the TV annulus (kappa value 0.91) and TV leaflets (kappa value 0.71) and moderate for the TV commissures (kappa value 0.59). As seen in Fig. 5, good interobserver correlations were found for measurement of TV annulus $(r=0.98, P<0.0001)$ and TV area $(r=0.95$, $P<0.0001)$ and fair correlation was found for TV commissural width $(r=0.51, P<0.001)$. In the same Figure, the interobserver agreement for TV annulus diameter (mean difference -0.28 $\pm 1.20 \mathrm{~mm}$, agreement: $2.12,-2.68$ ), for TV area (mean difference: $0.17 \pm 0.52 \mathrm{~cm}^{2}$, agreement: $1.21,-0.87)$, and for mean TV commissural width

Table 2 Normal (absolute and index) values of tricuspid valve annulus (diameter and area), Tricuspid valve area and the width of the 3 commissures

\begin{tabular}{|c|c|c|}
\hline Parameter & Absolute value & Index value \\
\hline $\begin{array}{l}\text { Tricuspid annulus } \\
\text { diameter }\end{array}$ & $4.0 \pm 0.7(\mathrm{~cm})$ & $2.2 \pm 0.4\left(\mathrm{~cm} / \mathrm{m}^{2}\right)$ \\
\hline $\begin{array}{l}\text { Tricuspid annulus } \\
\text { area }\end{array}$ & $10.0 \pm 2.9\left(\mathrm{~cm}^{2}\right)$ & $5.5 \pm 1.6\left(\mathrm{~cm}^{2} / \mathrm{m}^{2}\right)$ \\
\hline Tricuspid valve area & $4.8 \pm 1.6\left(\mathrm{~cm}^{2}\right)$ & $2.7 \pm 0.9\left(\mathrm{~cm}^{2} / \mathrm{m}^{2}\right)$ \\
\hline $\begin{array}{l}\text { Antero-septal } \\
\text { commissure }\end{array}$ & $5.4 \pm 1.5(\mathrm{~mm})$ & $2.9 \pm 0.8\left(\mathrm{~mm} / \mathrm{m}^{2}\right)$ \\
\hline $\begin{array}{c}\text { Postero-septal } \\
\text { commissure }\end{array}$ & $5.2 \pm 1.5(\mathrm{~mm})$ & $2.9 \pm 0.7\left(\mathrm{~mm} / \mathrm{m}^{2}\right)$ \\
\hline $\begin{array}{l}\text { Antero-posterior } \\
\text { commissure }\end{array}$ & $5.1 \pm 1.1(\mathrm{~mm})$ & $2.8 \pm 0.6\left(\mathrm{~mm} / \mathrm{m}^{2}\right)$ \\
\hline
\end{tabular}



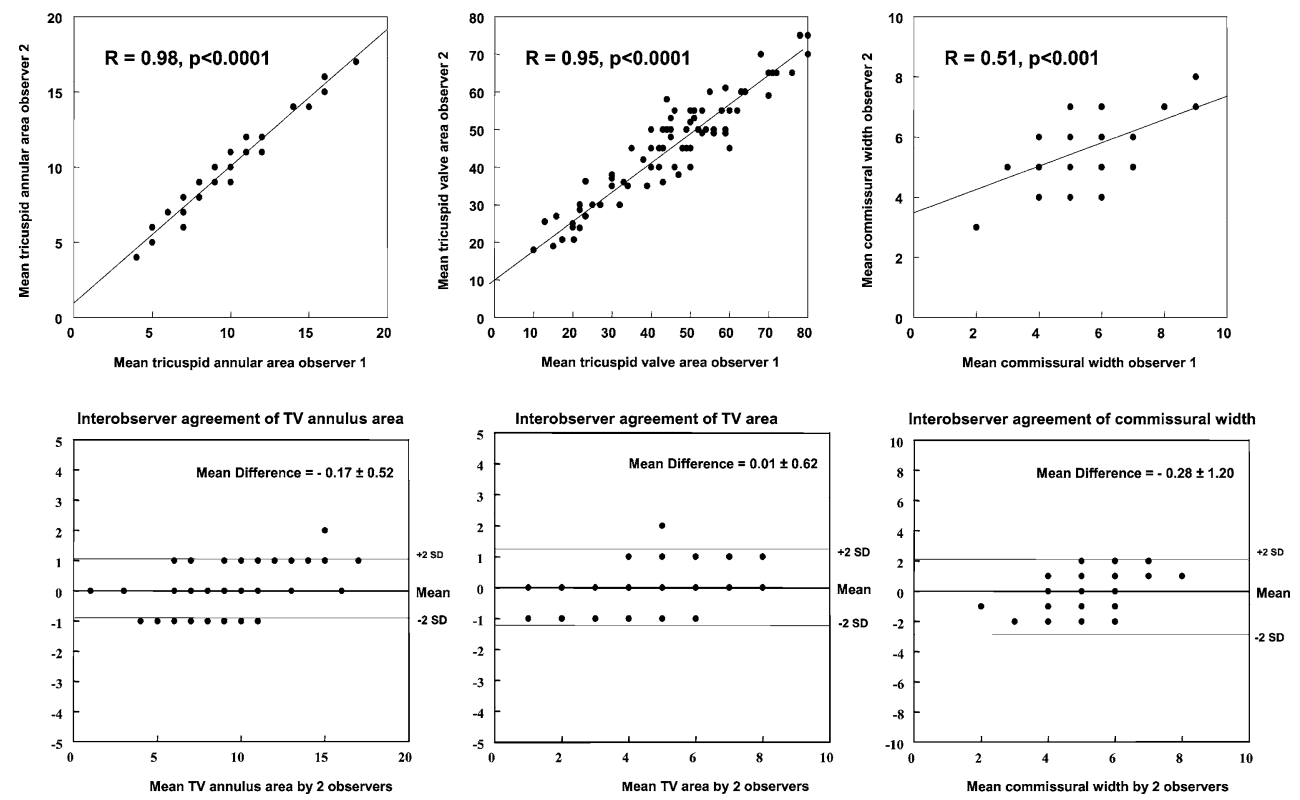

Fig. 5 Interobserver correlations (top) and Bland-Altman analysis (bottom) of TV annulus, leaflets, and commissures

(mean difference: $0.01 \pm 0.62 \mathrm{~mm}$, agreement: $1.25,-1.24)$ is displayed.

\section{Intraobserver variability}

The visualization score by the first observer at 2 separate settings was good for the TV annulus (kappa value 0.92) and TV leaflets (kappa value 0.73 ) and moderate for the TV commissures (kappa value 0.58). Intraobserver agreement was (mean difference $-0.26 \pm 1.15 \mathrm{~mm}$, agreement: $2.14,-2.56$ ) for TV annulus diameter, (mean difference: $0.15 \pm 0.50 \mathrm{~cm}^{2}$, agreement: 1.15 , -0.85 ) for TV area, and (mean difference: $0.02 \pm 0.60 \mathrm{~mm}$, agreement: $1.22,-1.18)$ for the mean TV commissural width.

\section{Discussion}

Two-dimensional echocardiography is a valuable imaging modality for the functional assessment of TV [11-13]. However, with 2DE it is not possible to visualize all TV cusps simultaneously in one cross-sectional view nor can detailed anatomical information of the TV annulus, leaflets, and commissures be provided. Previous studies and case reports described visualization of TV by RT3DE [9, 14] in abnormal states, while this study applied RT3DE for the morphological assessment of the normal TV anatomy. RT3DE allowed analysis of TV annulus, leaflets and commissures in the majority of patients. Beside this morphologic description, quantitative assessment could be obtained. However, it should be noticed that only patients with good 2DE image quality underwent RT3DE. In our experience these patients represent over $50 \%$ of the total number of patients referred to our echocardiographic laboratory. Nevertheless, RT3DE allowed TV analysis to a level quite comparable to that recently reported by others for the mitral valve leaflets [15].

One of the salient findings in our study was the identification of the TV leaflets as seen in the routine $2 \mathrm{DE}$ views. It is still a matter of controversy in echocardiographic textbooks. In one well known echocardiographic textbook [16], the leaflet seen in the apical 4-chamber view adjacent to the right ventricular free wall was described as being the anterior or posterior leaflet depending on the exact rotation and angulation of the image 


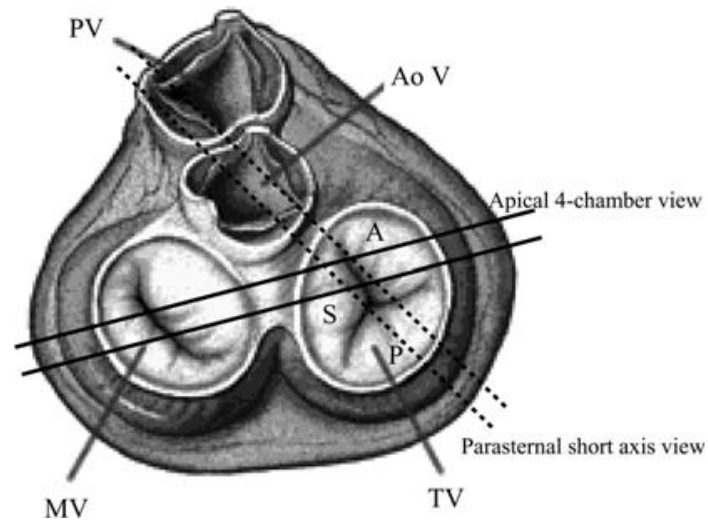

Fig. 6 Surgical view of the heart valves demonstrating the range of the two-dimensional echocardiographic 4-chamber and short-axis planes

plane. However, in our study this leaflet was consistently found to be the anterior leaflet (see Fig. 6 for explanation), as described in another textbook [17]. Also, in both these echocardiographic textbooks [16, 17] in the parasternal short-axis view the leaflet adjacent to the right ventricular free wall was described as being the anterior. However, as shown in Fig. 3 and explained in Fig. 6, in all patients in whom a leaflet could be identified in our study it was the posterior one.

In our study tricuspid annulus diameter (and area) could be reliably obtained with RT3DE. Tricuspid annulus measurement is of critical importance in the TV surgical decision-making process if a patient is operated for mitral valve disease and has concomitant TV regurgitation $[18,19]$. In addition, TV area could be reliably obtained and this may have important implica- tions for the diagnosis of tricuspid stenosis $[20,21]$. Visualization of commissures and measurement of its width were obtained with difficulty, in particular for the antero-posterior commissure. Commissural width also showed weak interobserver correlation. This may be due to differences in the commissural levels and tissue dropout. For proper assessment of the three commissures, more cut planes with different angles are needed. However, assessment of commissural width may also be a valuable tool for the diagnosis, follow up, and selection of therapeutic strategy of tricuspid stenosis. All our RT3DE measurements were consistent with the measurements described in anatomical studies [2, 3]. Our data may potentially take RT3DE a step further into clinical routine (providing accurate TV measurements) and may enhance the understanding of TV valve morphology during the cardiac cycle (Fig. 7). Also, the detailed assessment by RT3DE may affect the therapeutic decision of various TV abnormalities and thus expand the abilities of non-invasive cardiology [22].

\section{Limitation of study}

The main limitation of this study is that RT3DE data were not compared with a "gold standard" such as magnetic resonance imaging, autopsy or surgical findings. Also, RT3DE images more critically depend on 2DE image quality and could be obtained only in patients with sinus rhythm during hold breath that limits its application to all. The study included patients with narrow age range (21-39 years), and thus the normal findings
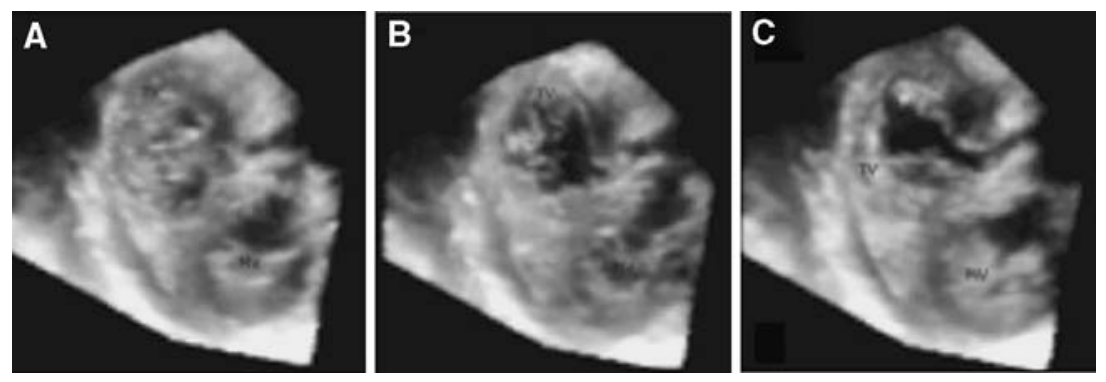

Fig. 7 Visualization of the 3 TV leaflets during valve closure (A), at early diastole (B), and at late diastole (C) 
in this study is confined to this age group and could not be applied to both extremities ( $<21$ and $>39$ years).

\section{Conclusion}

Three-dimensional imaging of the TV is feasible in a large number of patients. RT3DE may add to functional 2DE data in description of TV anatomy and providing highly reproducible and actual reality (anatomic and functional) measurements.

\section{References}

1. Lamers WH, Viragh S, Wessels A, Moorman A FM, Anderson RH (1995) Formation of the tricuspid valve in the human heart. Circulation 91:111-121

2. Silver MD, Lam JH, Ranganathan N, Wigle ED (1971) Morphology of the human tricuspid valve. Circulation 43:333-348

3. Wafae N, Hayashi H, Gerola LR, Vieira MC (1990) Anatomical study of the human tricuspid valve. Surg Radiol Anat 12:37-41

4. Maslow AD, Schwartz C, Singh AK (2004) Assessment of the tricuspid valve: a comparison of four transesophageal echocardiographic windows. J Cardiothorac Vasc Anesth 18:719-724

5. Pandian NG, Hsu TL, Schwartz SL, Weintraub A, Cao QL, Schneider AT, Gordon G, England M, Simonetti J (1992) Multiplane transesophageal echocardiography. Imaging planes, echocardiographic anatomy, and clinical experience with a prototype phased array OmniPlane probe. Echocardiography 9:649-666

6. Zaroff JG, Picard MH (2000) Transesophageal echocardiographic (TEE) evaluation of the mitral and tricuspid valves. Cardiol Clin 18:731-750

7. Light ED, Idriss SF, Wolf PD, Smith SW (2001) Realtime three-dimensional intracardiac echocardiography. Ultrasound Med Biol 27:1177-1183

8. Nekkanti R, Nanda NC, Ahmed S, Huang WY, Pacifico AD (2002) Transesophageal threedimensional echocardiographic demonstration of clefts in the anterior tricuspid valve leaflet. Am J Geriatr Cardiol 11:329-330

9. Schnabel R, Khaw AV, von Bardeleben RS, Strasser C, Kramm T, Meyer J, Mohr-Kahaly S (2005) Assessment of the tricuspid valve morphology by transthoracic real-time-3D-echocardiography. Echocardiography 22: $15-23$
10. Bland JM, Altman DG (1986) Statistical methods for assessing agreement between two methods of clinical measurement. Lancet 1:307-310

11. Colombo T, Russo C, Ciliberto GR, Lanfranconi M, Bruschi G, Agati S, Vitali E (2001) Tricuspid regurgitation secondary to mitral valve disease: tricuspid annulus function as guide to tricuspid valve repair. Cardiovasc Surg 9:369-377

12. Tunon J, Cordoba M, Rey M, Almeida P, Rabago R, Sanchez-Cascos A, Rabago P (1994) Assessment of chronic tricuspid regurgitation by colour Doppler echocardiography: a comparison with angiography in the catheterization room. Eur Heart J 15:1074-1084

13. Rivera JM, Mele D, Vandervoort PM, Morris E, Weyman AE, Thomas JD (1994) Effective regurgitant orifice area in tricuspid regurgitation: clinical implementation and follow-up study. Am Heart J 128:927-933

14. Faletra F, Marchesina U La, Bragato R, Chiara F De (2005) Three dimensional transthoracic echocardiography images of tricuspid stenosis. Heart 91:499

15. Sugeng L, Coon P, Weinert L, N Jolly N, Lammertin G, Bednarz JE, Thiele K, Lang RM (2006) Use of realtime 3-dimensional transthoracic echocardiography in the evaluation of mitral valve disease. J Am Soc Echocardiogr 19:413-421

16. Otto C (2004) Textbook of clinical echocardiography, 3rd edn. El-sevier, Saunders

17. Feigenbaum H (2005) Feigenbaum's Echocardiography, 6th edn. Lippincott, Williams \& Wilkins, Philadelphia, USA

18. Goldman ME, Guarino T, Fuster V, Mindich B (1987) The necessity for tricuspid valve repair can be determined intraoperatively by two-dimensional echocardiography. J Thorac Cardiovasc Surg 94:542550

19. De Simone R, Lange R, Tanzeem A, Gams E, Hagl S (1993) Adjustable tricuspid valve annuloplasty assisted by intraoperative transesophageal color Doppler echocardiography. Am J Cardiol 71:926-931

20. Ribeiro PA, al Zaibag M, Idris MT (1990) Percutaneous double balloon tricuspid valvotomy for severe tricuspid stenosis: 3-year follow-up study. Eur Heart J 11:1109-1112

21. Sharma S, Loya YS, Desai DM, Pinto RJ (1997) Percutaneous double-valve balloon valvotomy for multivalve stenosis: immediate results and intermediate-term follow-up. Am Heart J 133:64-70

22. Panza JA (2001) Real-time three-dimensional echocardiography: An overview. Int $\mathrm{J}$ Cardiovasc Imaging 17(3):227-235 